\title{
Rare cases of gas poisoning in the practice of forensic medicine
}

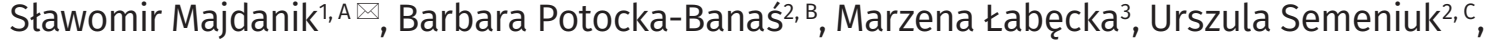 \\ Krzysztof Borowiak ${ }^{2, D}$ \\ ${ }^{1}$ Pomeranian Medical University in Szczecin, Department of Forensic Medicine, Powstańców Wlkp. 72, 70-111 Szczecin, Poland \\ 2 Pomeranian Medical University in Szczecin, Department of Clinical and Forensic Toxicology, Powstańców Wlkp. 72, 70-111 Szczecin, Poland \\ ${ }^{3}$ Poznan University of Medical Sciences, Department of Forensic Medicine, Fredry 10, 61-701 Poznań, Poland \\ A ORCID: 0000-0003-0472-1924; $\quad$ B ORCID: 0000-0003-0259-2006; C ORCID: 0000-0003-0247-4202; $\quad$ D ORCID: 0000-0002-2526-2686 \\ $\triangle$ majdanik@pum.edu.pl
}

\begin{abstract}
Cases of poisoning with gases other than carbon monoxide are rarely reported in forensic medicine. Usually they are associated with an accident, suicidal intoxication, or recreational inhalation to obtain psychoactive effects. The paper presents 2 cases of poisoning with an air freshener aerosol and argon. Both cases
\end{abstract}

were confirmed after confronting the results of post-mortem examination, additional tests, and the detailed circumstances of the incidents. These cases deserve attention because of both the clinical and scientific aspects. Keywords: air freshener; argon; nitrous oxide; death; poisoning.

\section{INTRODUCTION}

Gas poisoning is rarely encountered in forensic and toxicological practice. Most cases of poisoning result from exposure to carbon monoxide in a variety of circumstances, most often accidentally because of a malfunctioning of heating system, or suicide attempts. Cases of poisoning with other gases are described only occasionally due to the limited risk of exposure. The main causes of such intoxications are suicide, accidental exposure, or recreational drug use. Almost every case of such an exposure attracts the interest of court experts, and public opinion. This paper presents 2 cases of fatal gas poisonings that happened in unusual circumstances. One case of exposure (components of an air freshener) were associated with the deliberate actions of the deceased, and the 2 nd case was associated with accidental exposure to argon in the workplace.

\section{CASE 1}

The sudden death of a 15-year-old girl occurred while she was socializing with a group of her friends, at the home of one of them, after the intentional inhalation of an air freshener aerosol. Evidence showed that the girl had been intoxicating herself with air freshener aerosols and deodorants for several years. On the critical day, she fell over while doing this and hit her head on the floor. Paramedics called to the scene declared her dead without specifying the cause of death. The autopsy did not reveal any signs of injury, but features of generalized congestion of internal organs with ecchymoses on serous membranes and oedema of the brain and lungs were found. Toxicological tests of blood samples, urine and internal organs taken during the autopsy were negative for xenobiotics, including ethyl alcohol, narcotic substances, and organic solvents. Histopathological examination showed features of cerebral and pulmonary oedema, as well as features of acute pulmonary distension and recent myocardial injury. Based on this information, death by rapid asphyxia, resulting from a reactive bronchospasm triggered by the irritating effect of the inhaled aerosols, was declared. It was assumed that the death was directly caused by exposure to the components of the air freshener aerosol.

\section{CASE 2}

A fatal accident involving of a 45-year-old man took place on the construction site of a gas terminal. For unexplained reasons the worker, without adequate protective equipment, found himself inside a pipe filled with argon. While the man was being recovered and given first aid, another worker suffered a temporary loss of consciousness but survived. The autopsy of the deceased man revealed signs of medical procedures on the body, features of generalized congestion of internal organs with massive cerebral and pulmonary oedema. Characteristic features of acute myocardial hypoxia were found on histopathological examination. Biological material preserved during the autopsy were negative for ethyl alcohol and other organic compounds with potential pharmacological, psychotropic or intoxicating activity. Considering the results of the autopsy and the circumstances of the incident, the death of the 45-year-old man was attributed to rapid asphyxia resulting from hypoxia, which occurred in a situation of an excess of argon gas in the local air. 


\section{DISCUSSION}

The problem of recreational use of psychoactive substances has been known for a long time. Over the years there has been a marked change in the methods of intoxication and the number of substances used for this purpose. Interest in substances delivered by intravenous injection is decreasing, while oral and inhaled substances are becoming more popular. The widespread use of the Internet has caused a rapid increase in new ways of taking psychoactive substances and has also facilitated the sharing of experiences in this field. This relates to not just the most popular psychoactive substances, but also those less frequently used, such as nitrous oxide (commonly known as laughing gas) or substances contained in commercially available household products such as air fresheners, deodorants, gas for lighters, etc. $[1,2,3,4]$. Gases are very quickly absorbed from the lungs to adjacent tissues, which results in a rapid toxic effect and the onset of characteristic symptoms of intoxication. Moreover, exposure to chemically inert gases or those having irritant properties rapidly produces the effects of hypoxia and local effects on the respiratory tract.

Due to its anaesthetic and analgesic properties, nitrous oxide is used to induce and maintain general anaesthesia, as well as to achieve analgesic effects (e.g. during dressing changes, diagnostic tests) $[2,5,6,7]$. An overdose of nitrous oxide may lead to a variety of disorders, yet that has not prevented the widespread use of this gas in the food industry as a food additive and a foaming agent, especially in whipped cream spray. Nitrous oxide is listed under number E-942 in the database of food additives approved for use in the European Union. The list of food additives includes chemical substances regarded as safe and approved for use [2, 8].

Air fresheners contain many organic compounds, including hydrocarbons, polyols, aldehydes, derivatives of aromatic hydrocarbons, etc. These substances are used because of the potential narcotic effect, and they also have irritating, allergenic (may trigger asthma attacks), carcinogenic and teratogenic properties [9]. Importantly, in high concentrations, these gases cause hypoxia. The use of these substances by adolescents results from their easy and legal availability, as well as low price $[4,10]$.

Argon is a noble gas found only in trace concentrations in the natural environment. In industry it is used, for instance, as a shielding gas during welding. In the process of welding pipes, the joined elements are sealed with special tampon plugs to prevent the leakage of argon, and during work the welder should wear an oxygen mask for protection. Argon also has other applications, e.g. for filling light bulbs, glass units or computer hard drives. Argon is a chemically inert and non-toxic gas, but its increased concentrations in the air cause a decrease in oxygen levels, which may result in hypoxia symptoms $[11,12]$.

\section{SUMMARY}

The described cases of gas poisoning are rare in toxicological and forensic practice. Diagnosis of death in such cases, when information about the circumstances is unavailable, may be impossible. Interestingly, in our cases, routine autopsy did not reveal any characteristic signs that could be attributed to earlier exposure to a specific gaseous compound. Features of generalized hyperemia or acute hypoxia identified in our cases may occur in various circumstances, even by natural causes associated with disease. Moreover, routine toxicological tests are also negative in such cases. Therefore, when investigating cases of death by gas poisoning, close cooperation between the physicians or toxicologists and policemen and lawyers are particularly important to determine the exact circumstances of the death, and consequently the actual cause of death. In addition, the presented cases are rarely encountered in forensic medicine, which makes them interesting from both clinical and scientific aspects.

\section{REFERENCES}

1. Musshoff F, Hagemeier L, Kirschbaum K, Madea B. Two cases of suicide by asphyxiation due to helium and argon. Forensic Sci Int 2012;223 (1-3):e27-30.

2. Potocka-Banaś B, Majdanik S, Dutkiewicz G, Borowiak K, Janus T. Death caused by addictive inhalation of nitrous oxide. Hum Exp Toxicol 2011;30(11):1875-7.

3. Weimann J. Toxicity of nitrous oxide. Best Pract Res Clin Anaesthesiol 2003;17(1):47-61.

4. Kim S, Hong SH, Bong CK, Cho MH. Characterization of air freshener emission: the potential health effects. J Toxicol Sci 2015;40(5):535-50.

5. Baum JA. The carrier gas in anaesthesia: nitrous oxide/oxygen, medical air/oxygen and pure oxygen. Curr Opin Anaesthesiol 2004;17(6):513-6.

6. Myles PS, Leslie K, Chan MT, Forbes A, Paech MJ, Peyton P, et al. Avoidance of nitrous oxide for patients undergoing major surgery: a randomized controlled trial. Anesthesiology 2007;107(2):221-31.

7. Sanders RD, Weimann J, Maze M. Biologic effects of nitrous oxide: a mechanistic and toxicologic review. Anesthesiology 2008;109(4):707-22.

8. European Parliament and Council Directive No 95/2/EC of 20 February 1995 on food additives other than colours and sweeteners (OJ No L 61, 18. 3. 1995, p. 1).

9. Steinemann A. Fragranced consumer products: effects on asthmatics. Air Qual Atmos Health 2018;11(1):3-9.

10. Hitosugi M, Tsukada C, Yamauchi S, Matsushima K, Furukawa S, Morita S, et al. An autopsy case of fatal repellent air freshener poisoning. Leg Med (Tokyo) 2015;17(5):360-3.

11. Grassberger M, Krauskopf A. Suicidal asphyxiation with helium: report of three cases. Wien Klin Wochenschr 2007;119(9-10):323-5.

12. Sandomirsky M, Crifasi JA, Long C, Mitchell EK. Case report of fatal complication in prostatic cryotherapy. First reported death due to argon gas emboli. Am J Forensic Med Pathol 2012;33(1):68-72 